\title{
ON A CONJECTURE OF TARSKI ON PRODUCTS OF CARDINALS
}

\author{
THOMAS JECH AND SAHARON SHELAH
}

(Communicated by Andreas R. Blass)

\begin{abstract}
We look at an old conjecture of A. Tarski on cardinal arithmetic and show that if a counterexample exists, then there exists one of length $\omega_{1}+\omega$.
\end{abstract}

In the early days of set theory, Hausdorff and Tarski established basic rules for exponentiation of cardinal numbers. In [T] Tarski showed that for every limit ordinal $\beta, \prod_{\xi<\beta} \aleph_{\xi}=\aleph_{\beta}^{|\beta|}$, and conjectured that

$$
\prod_{\xi<\beta} \aleph_{\sigma_{\xi}}=\aleph_{\alpha}^{|\beta|}
$$

holds for every ordinal $\beta$ and every increasing sequence $\left\{\sigma_{\xi}\right\}_{\xi<\beta}$ such that $\lim _{\xi<\beta} \sigma_{\xi}=\alpha$. He remarked that (1) holds for every countable ordinal $\beta$.

Remarks. 1. The left-hand side of $(1)$ is less than or equal to the right-hand side.

2. If $\beta$ has $|\beta|$ disjoint cofinal subsets then the equality (1) holds. Thus the first limit ordinal that can be the length of a counterexample to (1) is $\omega_{1}+\omega$. Proof. Let $\left\{A_{i}: i<|\beta|\right\}$ be disjoint cofinal subsets of $\beta$. Then $\prod_{\xi<\beta} \aleph_{\sigma_{\xi}} \geq$ $\prod_{i<|\beta|} \prod_{\xi \in A_{i}} \aleph_{\sigma_{\xi}} \geq \prod_{i<|\beta|} \aleph_{\alpha}=\aleph_{\alpha}^{|\beta|}$.

It is not difficult to see that if one assumes the singular cardinals hypothesis then (1) holds. With the hindsight given by results obtained in the last twenty years, it is also not difficult to find a counterexample to Tarski's conjecture. For instance, using the model described in [M], one can have an increasing sequence of cardinals of length $\beta=\omega_{1}+\omega$ whose product does not satisfy (1). The purpose of this note is to show that if Tarski's conjecture fails then it fails in

Received by the editors June 8, 1989 and, in revised form, September 21, 1990.

1980 Mathematics Subject Classification (1985 Revision). Primary 03E00.

Key words and phrases. Cardinal arithmetic, singular cardinals problem, $p c f$.

The first author was supported partially by an NSF grant. I wish to express my gratitude to the Mathematical Institute of the Eidgenössische Technische Hochschule in Zürich for its hospitality during my visit.

The second author was supported partially by the B.S.F; publication \#385. 
this specific way. Namely, if there is a counterexample then there is one of length $\omega_{1}+\omega$.

The main result of this paper is the following:

Theorem. A necessary and sufficient condition for Tarski's conjecture to fail is the existence of a singular cardinal $\aleph_{\gamma}$ of cofinality $\aleph_{1}$ such that $\aleph_{\gamma}>\aleph_{\omega_{1}}{ }_{1}$ and $\aleph_{\gamma} \aleph_{1}>\aleph_{\gamma+\omega} \aleph_{0}$.

If $\aleph_{\gamma}$ is a cardinal that satisfies the condition then the sequence $\left\{\aleph_{\xi}\right\}_{\xi<\omega_{1}} \cup$ $\left\{\aleph_{\gamma+n}\right\}_{n<\omega}$ is a counterexample to (1):

$$
\prod_{\xi<\omega_{1}} \aleph_{\xi} \cdot \prod_{n<\omega} \aleph_{\gamma+n}=\aleph_{\omega_{1}}^{\aleph_{1}} \cdot \aleph_{\gamma+\omega}{ }^{\aleph_{0}}<\aleph_{\gamma+\omega}{ }^{\left|\omega_{1}+\omega\right|} .
$$

Such a cardinal exists in one of Magidor's models, e.g. when $\aleph_{\gamma}=\aleph_{\omega_{1}+\omega_{1}}$ is a strong limit, $\aleph_{\omega_{1}+\omega_{1}} \aleph_{1}=\aleph_{\omega_{1}+\omega_{1}+\omega+2}$ and $\aleph_{\omega_{1}+\omega_{1}+\omega}{ }^{N_{0}}=\aleph_{\omega_{1}+\omega_{1}+\omega+1}$.

Also, if $\lambda>\aleph_{\omega_{1}}$ is a strong limit singular cardinal of cofinality $\aleph_{1}$ such that $\lambda^{\aleph_{1}}>\lambda^{+\left(2^{N_{0}}\right)^{+}}$then we have a counterexample as $\left(\lambda^{+\omega}\right)^{\aleph_{0}}<\lambda^{+\left(2^{N_{0}}\right)^{+}}$(by [ShA2, Chapter XIII, 5.1]).

The rest of this paper is devoted to the proof that the condition is necessary.

Assume that Tarski's conjecture fails, and let $\beta$ be a limit ordinal for which there exists a sequence $\left\{\sigma_{\xi}\right\}_{\xi<\beta}$ that gives a counterexample:

$$
\prod_{\xi<\beta} \aleph_{\sigma_{\xi}}<\aleph_{\alpha}{ }^{\kappa},
$$

where

$$
\kappa=|\beta| \text { and } \alpha=\lim _{\xi<\beta} \sigma_{\xi} .
$$

Lemma 1. If (2) holds then $\operatorname{cf} \beta<\kappa<\beta$, and there exists an ordinal $\gamma<\alpha$ such that $\aleph_{\gamma}{ }^{\kappa}>\aleph_{\alpha}$.

Proof. If (2) holds then $\beta$ does not have $|\beta|$ disjoint cofinal subsets, and it follows that $\beta$ is not a cardinal, and that $\operatorname{cf} \beta<|\beta|$.

Assuming that $\aleph_{\gamma}{ }^{\kappa} \leq \aleph_{\alpha}$ holds for all $\gamma<\alpha$, we pick a cofinal sequence $\left\{\alpha_{i}\right\}_{i<\mathrm{cf} \beta}$ with limit $\alpha$, and then

$$
\aleph_{\alpha}{ }^{\kappa}=\left(\sum_{i<\mathrm{cf} \beta} \aleph_{\alpha_{i}}\right)^{\kappa} \leq \prod_{i<\mathrm{cf} \beta} \aleph_{\alpha_{i}}{ }^{\kappa} \leq \prod_{i<\mathrm{cf} \beta} \aleph_{\alpha}=\aleph_{\alpha}{ }^{\mathrm{cf} \beta}=\prod_{i<\mathrm{cf} \beta} \aleph_{\alpha_{i}} \leq \prod_{\xi<\beta} \aleph_{\sigma_{\xi}},
$$

contrary to $(2)$.

Now consider the shortest counterexample to Tarski's conjecture.

Lemma 2. If $\beta$ is the least ordinal for which (2) holds then $\beta=\kappa+\omega$ where $\kappa$ is an uncountable cardinal.

Proof. Without loss of generality, the sequence $\sigma$ is continuous. (We can replace each $\sigma_{\xi}$ by the limit of the sequence at $\xi$, for each limit ordinal $\xi$.) 
Let $\kappa=|\beta|$. We claim that for every limit ordinal $\eta<\beta, \aleph_{\sigma_{\eta}}{ }^{\kappa}<\aleph_{\alpha}$. If this were not true then, because $\beta>\kappa$, there would be a limit ordinal $\eta$ such that $\kappa \leq \eta<\beta$ and that $\aleph_{\sigma_{\eta}}\left|\sigma_{\eta}\right| \geq \aleph_{\alpha}{ }^{\kappa}>\prod_{\xi<\eta} \aleph_{\sigma_{\xi}}$, which would make the sequence $\left\{\sigma_{\xi}\right\}_{\xi<\eta}$ a counterexample to Tarski's conjecture as well, contrary to the minimality of $\beta$.

Thus $\beta=\delta+\omega$ for some limit ordinal $\delta$. It is clear that the sequence

$$
\left\{\aleph_{\sigma_{\xi}}: \xi \leq \kappa \text { or } \xi>\delta\right\}
$$

of length $\kappa+\omega$ is also a counterexample, and by the minimality of $\beta$ we have $\beta=\kappa+\omega$.

Now consider the least ordinal $\gamma$ such that $\kappa_{\gamma}{ }^{k}>\aleph_{\alpha}$. We shall show that $\operatorname{cf} \gamma=\kappa$ (and so $\kappa$ is a regular uncountable cardinal). We also establish other properties of $\aleph_{\gamma}$.

Lemma 3. If Tarski's conjecture fails, then there is a cardinal $\aleph_{\gamma}$ of uncountable cofinality $\kappa$ such that $\gamma>\kappa$, and that

$$
\begin{gathered}
\text { for every } \nu<\gamma, \quad \aleph_{\nu}{ }^{\kappa}<\aleph_{\gamma}, \\
\aleph_{\gamma}{ }^{\kappa}>\aleph_{\gamma+\omega}{ }^{\aleph_{0}} .
\end{gathered}
$$

Proof. Let $\beta=\kappa+\omega$ be the least ordinal for which (2) holds, for some increasing continuous sequence $\left\{\sigma_{\xi}: \xi<\beta\right\}$ with limit $\alpha$, and let $\gamma$ be the least ordinal such that $\aleph_{\gamma}{ }^{\kappa}>\aleph_{\alpha}$.

First we observe that for every $\nu<\gamma, \aleph_{\nu}{ }^{\kappa}<\aleph_{\gamma}$. This is because if $\aleph_{\nu}{ }^{\kappa} \geq \aleph_{\gamma}$ then $\aleph_{\nu}{ }^{\kappa} \geq \aleph_{\gamma}{ }^{\kappa}>\aleph_{\alpha}$, contradicting the minimality of $\gamma$.

As a consequence, we have $c f \gamma \leq \kappa$ : otherwise, we would have $\kappa_{\gamma}{ }^{\kappa}=$ $\sum_{\nu<\gamma} \aleph_{\nu}{ }^{\kappa}=\aleph_{\gamma}<\aleph_{\alpha}$, a contradiction. Also, if $\gamma=\lim _{i \rightarrow c f \gamma} \gamma_{i}$, then $\aleph_{\gamma}{ }^{\kappa}=$ $\left(\sum_{i<\mathrm{cf} \gamma} \aleph_{\gamma_{i}}\right)^{\kappa} \leq \prod_{i<\mathrm{cf} \gamma} \aleph_{\gamma_{i}}{ }^{\kappa} \leq \prod_{i<\mathrm{cf} \gamma} \aleph_{\gamma}=\aleph_{\gamma}{ }^{\mathrm{cf} \gamma}$ and so we have

$$
\aleph_{\gamma}{ }^{\mathrm{cf} \gamma}=\aleph_{\gamma}{ }^{\kappa} \text {. }
$$

Since $\aleph_{\alpha}<\aleph_{\gamma}{ }^{\kappa}$, we have $\aleph_{\alpha}{ }^{\kappa} \leq \aleph_{\gamma}{ }^{\kappa}=\aleph_{\gamma}{ }^{\text {cf } \gamma} \leq \aleph_{\alpha}{ }^{\text {cf } \gamma}$, and so $\aleph_{\alpha}{ }^{\text {cf } \gamma}=\aleph_{\alpha}{ }^{\kappa}$, and $\aleph_{\alpha}{ }^{\text {cf } \gamma}>\prod_{\xi<\beta} \aleph_{\sigma_{\xi}}$. Hence the sequence

$$
\left\{\aleph_{\sigma_{\xi}}: \xi \leq \mathrm{cf} \gamma \text { or } \xi>\kappa\right\}
$$

of length $\mathrm{cf} \gamma+\omega$ is also a counterexample, and it follows that $\kappa=\mathrm{cf} \gamma$.

For every limit $\eta<\beta$ we have $\aleph_{\sigma_{\eta}}{ }^{k}<\aleph_{\alpha}$, and in particular $\aleph_{\sigma_{\kappa}}{ }^{\kappa}<\aleph_{\alpha}$. Since $\aleph_{\gamma}{ }^{\kappa}>\aleph_{\alpha}$, we have $\gamma>\kappa$. Finally,

$$
\prod_{\xi<\beta} \aleph_{\sigma_{\xi}}=\prod_{\xi<\kappa} \aleph_{\sigma_{\xi}} \cdot \prod_{n<\omega} \aleph_{\sigma_{\kappa+n}}=\aleph_{\sigma_{\kappa}}{ }^{\kappa} \cdot \aleph_{\alpha}{ }^{N_{0}}=\aleph_{\alpha}{ }^{\aleph_{0}},
$$


and because $\aleph_{\gamma}{ }^{\kappa}=\aleph_{\alpha}{ }^{\kappa}>\prod_{\xi<\beta} \aleph_{\sigma_{\xi}}$, we have $\aleph_{\gamma}{ }^{\kappa}>\aleph_{\alpha}{ }^{\aleph_{0}}$. Since $\alpha=\underset{n \rightarrow \omega}{\lim \sigma_{\kappa+n}} \geq$ $\gamma+\omega$, we have

completing the proof.

$$
\aleph_{\gamma}^{\kappa}>\aleph_{\gamma+\omega}^{\aleph_{0}}
$$

The cardinal $\aleph_{\gamma}$ obtained in Lemma 3 satisfies all the conditions stated in the theorem except for the requirement that its cofinality be $\aleph_{1}$. Thus the following lemma will complete the proof.

Lemma 4. Let $\aleph_{\gamma}$ be a singular cardinal of cofinality $\kappa>\aleph_{1}$ such that $\gamma>\kappa$ and that

$$
\text { for every } \nu<\gamma, \quad \aleph_{\nu}{ }^{\kappa}<\aleph_{\gamma} .
$$

Assume further that for every $\delta, \omega_{1}<\delta<\gamma$, of cofinality $\aleph_{1}$,

$$
\text { if for every } \nu<\delta, \aleph_{\nu}{ }^{\aleph_{1}}<\aleph_{\delta} \text {, then } \aleph_{\delta}{ }^{\aleph_{1}} \leq \aleph_{\delta+\omega}{ }^{\aleph_{0}} \text {. }
$$

Then $\aleph_{\gamma}{ }^{\kappa} \leq \aleph_{\gamma+\omega}{ }^{\kappa_{0}}$.

Lemma 4 implies that the least $\gamma$ in Lemma 3 has cofinality $\aleph_{1}$, and the theorem follows. The rest of the paper is devoted to the proof of Lemma 4. We use the second author's analysis of pcf.

Definition. If $A$ is a set of regular cardinals, let

$$
\Pi A=\{f: \operatorname{dom} f=A \text { and } f(\lambda)<\lambda \text { for all } \lambda \in A\} .
$$

If $I$ is an ideal on $A$ then $\Pi A / I$ is a partially ordered set under

$$
f \leq_{I} g \text { iff }\{\lambda: f(\lambda)>g(\lambda)\} \in I,
$$

and similarly for filters on $A$. If $D$ is an ultrafilter on $A$, then $\Pi A / D$ is a linearly ordered set, and $\operatorname{cf}(\Pi A / D)$ denotes its cofinality. Let

$$
\operatorname{pcf}(A)=\{\operatorname{cf}(\Pi A / D): D \text { an ultrafilter on } A\} .
$$

It is clear that

$$
\begin{array}{r}
A \subseteq \operatorname{pcf}(A), A_{1} \subseteq A_{2} \quad \text { implies } \operatorname{pcf}\left(A_{1}\right) \subseteq \operatorname{pcf}\left(A_{2}\right), \quad \text { and } \\
\operatorname{pcf}\left(A_{1} \cup A_{2}\right)=\operatorname{pcf}\left(A_{1}\right) \cup \operatorname{pcf}\left(A_{2}\right),
\end{array}
$$

and it is not difficult to show (using ultrapowers of ultrapowers) that

$$
\begin{aligned}
& \text { if }|\operatorname{pcf}(A)|<\min A, \quad \text { then } \operatorname{pcf}(\operatorname{pcf}(A))=\operatorname{pcf}(A) \text { and } \\
& \operatorname{pcf}(A) \text { has a greatest element. }
\end{aligned}
$$

Theorem (Shelah [Sh345]). If $2^{|A|}<\min (A)$ then there exists a family $\left\{B_{\nu}\right.$ : $\nu \in \operatorname{pcf}(A)\}$ of subsets of $A$ such that

(7) for every ultrafilter $D$ on $A, \operatorname{cf}(\Pi A / D)=$ the least $\nu$ such that $B_{\nu} \in D$. 
For every $\lambda \in \operatorname{pcf}(A)$ there exists a family $\left\{f_{\alpha}: \alpha<\lambda\right\} \subseteq \Pi A$ such that

$\alpha<\beta$ implies $f_{\alpha}<f_{\beta} \bmod J_{<\lambda}$, where $J_{<\lambda}$ is the ideal generated by $\left\{B_{\nu}: \nu<\lambda\right\}$, and the $f_{\alpha}$ 's are cofinal in $\Pi B_{\lambda} \bmod J_{<\lambda}$.

An immediate consequence of (7) is that $|\operatorname{pcf}(A)| \leq 2^{|A|}$. The sets $B_{\nu}$ $(\nu \in \operatorname{pcf}(A))$ are called generators for $A$. Note that $\max B_{\nu}=\nu$ when $\nu \in A$, and that $\max \left(\operatorname{pcf}\left(B_{\nu}\right)\right)=\nu$ for all $\nu$.

We shall use some properties of generators.

Lemma 5 [Sh345]. Let $B_{\nu}$ be generators for $A$. For every $X \subseteq A$ there exists a finite set $F \subseteq \operatorname{pcf}(X)$ such that $X \subseteq \bigcup\left\{B_{\nu}: \nu \in F\right\}$.

Proof. Let $Y=\operatorname{pcf}(X)$, and assume that the lemma fails. Then $\left\{X-B_{\nu}\right.$ : $\nu \in Y\}$ has the finite intersection property and so there is an ultrafilter $D$ on $A$ such that $X \in D$ and $B_{\nu} \notin D$ for all $\nu \in Y$. Let $\mu=\operatorname{cf}(\Pi A / D)$. Then $\mu \in \operatorname{pcf}(X)$ and by (7), $B_{\mu} \in D$. A contradiction.

For each $X \subseteq A$, let $s(X)$ (a support of $X$ ) denote a finite set $F \subseteq \operatorname{pcf}(X)$ with the property that $X \subseteq \bigcup_{\nu \in F} B_{\nu}$.

The set $\operatorname{pcf}(A)$ has a set of generators that satisfy a transitivity condition:

Lemma 6 [Sh345]. Assume that $2^{|A|}<\min (A)$ and let $\bar{A}=\operatorname{pcf}(A)$. Then $\operatorname{pcf}(\bar{A})=\bar{A}$ and $\bar{A}$ has a set of generators $\left\{B_{\nu}: \nu \in \bar{A}\right\}$ that satisfy, in addition to $(7)$,

$$
\text { if } \xi \in B_{\nu} \text { then } B_{\xi} \subseteq B_{\nu} \text {. }
$$

We use the transitivity to prove the next lemma.

Lemma 7. Assume that $2^{|A|}<\min (A)$, let $\bar{A}=\operatorname{pcf}(A)$, let $B_{\nu}, \nu \in \bar{A}$, be transitive generators for $\bar{A}$, and for each $X \subseteq \bar{A}$ let $s(X)$ be a support of $X$. If $A=\bigcup_{i \in I} A_{i}$, then

$$
\bar{A}=\bigcup\left\{\operatorname{pcf}\left(B_{\nu}\right): \nu \in \operatorname{pcf}\left(\bigcup_{i \in I} s\left(\operatorname{pcf}\left(A_{i}\right)\right)\right)\right\} .
$$

Corollary. $\max (\bar{A})=\max \operatorname{pcf} \bigcup_{i \in I} s\left(\operatorname{pcf}\left(A_{i}\right)\right)$.

Proof of corollary. Let $\lambda=\max (\bar{A}) ; \lambda \in \operatorname{pcf}\left(B_{\nu}\right)$ for some $\nu$ in $\operatorname{pcf}\left(\bigcup_{i} s\left(A_{i}\right)\right)$. Since $\max \left(\operatorname{pcf}\left(B_{\nu}\right)\right)=\nu$, we have $\lambda \leq \nu$.

Proof. Let $X=\bigcup_{i \in I} s\left(\operatorname{pcf}\left(A_{i}\right)\right)$ and $F=s(X)$. We have

$$
\begin{aligned}
A & =\bigcup_{i \in I} A_{i} \subseteq \bigcup_{i \in I} \operatorname{pcf}\left(A_{i}\right) \subseteq \bigcup_{i \in I}\left\{B_{\xi}: \xi \in s\left(\operatorname{pcf}\left(A_{i}\right)\right)\right\} \\
& =\bigcup\left\{B_{\xi}: \xi \in X\right\} \subseteq \bigcup\left\{B_{\xi}: \xi \in \bigcup_{\nu \in F} B_{\nu}\right\} \subseteq \bigcup_{\nu \in F} B_{\nu}
\end{aligned}
$$


(the last inclusion is a consequence of transitivity (9)). Therefore

$$
\bar{A}=\operatorname{pcf}(A) \subseteq \operatorname{pcf}\left(\bigcup_{\nu \in F} B_{\nu}\right)=\bigcup_{\nu \in F} \operatorname{pcf}\left(B_{\nu}\right) \subseteq \bigcup\left\{\operatorname{pcf}\left(B_{\nu}\right): \nu \in \operatorname{pcf}(X)\right\} .
$$

Toward the proof of Lemma 4, let $\left\{\gamma_{i}: i<\kappa\right\}$ be a continuous increasing sequence of limit ordinals of cofinality $<\kappa$, such that $\lim _{i \rightarrow \kappa} \gamma_{i}=\gamma, 2^{\kappa}<\aleph_{\gamma_{0}}$, and that for all $i<\kappa$,

$$
\text { for all } \nu<\gamma_{i}, \quad \aleph_{\nu}{ }^{\kappa}<\aleph_{\gamma_{i}} \text {. }
$$

Lemma 8. There is a closed unbounded set $C \subseteq \kappa$ such that for all $n=$ $1,2, \ldots$,

$$
\max \operatorname{pcf}\left(\left\{\aleph_{\gamma_{i}+n}: i \in C\right\}\right) \leq \aleph_{\gamma+n} .
$$

Proof. We show that for each $n$ there exists a closed unbounded set $C_{n} \subseteq \kappa$ such that $\max \operatorname{pcf}\left(\left\{\aleph_{\gamma_{i}+n}: i \in C_{n}\right\}\right) \leq \aleph_{\gamma+n}$. To prove this, let $n \geq 1$ be fixed and let $A=\left\{\aleph_{\gamma_{i}+n}: i<\kappa\right\}$. Let $\lambda$ be the least element of $\operatorname{pcf}(A)$ above $\aleph_{\gamma+n}$ (if there is none there is nothing to prove). Let $\left\{B_{\nu}: \nu \in \operatorname{pcf}(A)\right\}$ be subsets of $A$ that satisfy (7), and let $\left\{S_{\nu}: \nu \in \operatorname{pcf}(A)\right\}$ be the subsets of $\kappa$ such that $B_{\nu}=\left\{\aleph_{\gamma_{i}+n}: i \in S_{\nu}\right\}$. It suffices to prove that the set $S_{\aleph_{\gamma+1}} \cup \cdots \cup S_{\aleph_{\gamma+n}}$ contains a closed unbounded set.

Thus assume that the set $S=\kappa-\left(S_{\aleph_{\gamma+1}} \cup \cdots \cup S_{\aleph_{\gamma+n}}\right)$ is stationary. Let $J_{<\lambda}$ be the ideal on $A$ generated by $\left\{B_{\nu}: \nu<\lambda\right\}$. By Shelah's theorem there exists a family $\left\{f_{\alpha}: \alpha<\lambda\right\}$ in $\Pi A$ such that $\alpha<\beta$ implies $f_{\alpha}<f_{\beta} \bmod J_{<\lambda}$. Since all the sets $B_{\nu}, \nu<\aleph_{\gamma}$, are bounded, we get a family $\left\{g_{\alpha}: \alpha<\lambda\right\}$ of functions on $S$ such that $g_{\alpha}(i)<\aleph_{\gamma_{i}+n}$ for all $i \in S$, and such that $\alpha<\beta$ implies that $g_{\alpha}(i)<g_{\beta}(i)$ for eventually all $i \in S$. This contradicts the results in [GH] by which, under the assumption (5), any family of almost disjoint functions in $\prod_{i \in S} \aleph_{\gamma_{i}+n}$ has size at most $\aleph_{\gamma+n}$.

Proof of Lemma 4. Let $\gamma$ be a singular cardinal of cofinality $\kappa>\aleph_{1}$ that satisfies (5) and (6). Let $\lambda$ be a regular cardinal such that $\aleph_{\gamma}<\lambda \leq \aleph_{\gamma}{ }^{k}$. We shall prove that $\lambda \leq \aleph_{\gamma+\omega} \aleph_{0}$.

Let $\left\{\gamma_{i}: i<\kappa\right\}$ be an increasing continuous sequence that satisfies (10), and let $C$ be a closed unbounded subset of $\kappa$ given by Lemma 8 . Let

$$
S=\left\{i \in C: c f \gamma_{i}=\aleph_{1}\right\} .
$$

As $\kappa \geq \aleph_{2}, S$ is a stationary subset of $\kappa$.

Lemma 9. There exist regular cardinals $\lambda_{i}, i \in S$, such that for each $i \in S$, $\aleph_{\gamma_{i}}<\lambda_{i} \leq \aleph_{\gamma_{i}}{ }^{N_{1}}$, and an ultrafilter $D$ on $S$ such that $\operatorname{cf}\left(\prod_{i \in S} \lambda_{i} / D\right)=\lambda$.

Proof. Let $I_{0}$ be the nonstationary ideal on $S$. There are $\lambda$ cofinal subsets $X$ of $\omega_{\gamma}$ of size $|X|=\kappa$. For every such set $X$, let $F_{X} \in \prod_{i \in C}\left[\aleph_{\gamma_{i}}\right]^{\leq \kappa}$ be 
the function defined by $F(i)=X \cap \omega_{\gamma_{i}}$. Then when $X \neq Y, F_{X}$ and $F_{Y}$ are eventually distinct.

For every $i \in S$ we have $\aleph_{\gamma_{i}}{ }^{k}=\aleph_{\gamma_{i}}{ }^{\kappa_{1}}$ (by (10)), and so there exist $\lambda I_{0^{-}}$ distinct functions in $\prod_{i \in S} \mathcal{N}_{\gamma_{i}}{ }^{\aleph_{1}}$. [ $f$ and $g$ are $I_{0}$-distinct if $\{i: f(i)=g(i)\} \in$ $I_{0}$.]

Consider the partial ordering $f<_{I_{0}} g$ defined by $\{i: f(i) \geq g(i)\} \in I_{0}$; since $I_{0}$ is $\sigma$-complete, $<_{I_{0}}$ is well-founded. Let $g$ be a $<_{I_{0}}$-minimal function with the property that $g(i) \leq \aleph_{\gamma_{i}}{ }^{\kappa_{1}}$ and that there are $\lambda I_{0}$-distinct functions below $g$.

Let $I$ be the extension of $I_{0}$ generated by all the stationary subsets $X$ of $S$ that have the property that $g$ is not minimal on $I_{0}[X]$ (i.e. there is a function $g^{\prime}$ such that $g^{\prime}(i)<g(i)$ almost everywhere on $X$ and below $g^{\prime}$ there are $\lambda$ $I_{0}$-distinct functions).

Claim. $I$ is a normal $\kappa$-complete ideal on $S$.

Proof. Let $X_{i}, i<\kappa$, be sets in $I$, and let for each $i<\kappa, g_{i}<g$ on $X_{i}$ and $\left\langle h_{\xi}^{i}: \xi<\lambda\right\rangle$ witness that $X_{i} \in I$. Then one constructs witnesses $\bar{g}$ and $\left\langle\bar{h}_{\xi}: \xi<\lambda\right\rangle$ for $X=\left\{j \in \kappa: j \in \bigcup_{i<j} X_{i}\right\}$ by letting $\bar{g}(j)=g_{i}(j)$ and $\bar{h}_{\xi}(j)=h_{\xi}^{i}(j)$ where $i$ is some $i<j$ such that $j \in X_{i}$.

For example, let us show that $\bar{h}_{\xi}$ and $\bar{h}_{\eta}$ are $I_{0}$-distinct if $\xi \neq \eta$. Assume that $\bar{h}_{\xi}=\bar{h}_{\eta}$ on a stationary subset $S_{1}$ of $S$. Then on a stationary subset $S_{2}$ of $S_{1}$ the $i$ less than $j \in S_{2}$ chosen such that $j \in X_{i}$ is the same $i$, and we have $h_{\xi}^{i}=h_{\eta}^{i}$ on $S_{2}$, a contradiction.

Let $\left\{h_{\xi}: \xi<\lambda\right\}$ be a family of $I_{0}$-distinct functions below $g$.

Claim. For every $h<_{I} g$ there is some $\xi_{0}<\lambda$ such that for all $\xi \geq \xi_{0}$, $h<_{I} h_{\xi}$.

Proof. If there are $\lambda$ many $\xi$ 's such that $h \geq h_{\xi}$ on an $I$-positive set, then (because $2^{\kappa}<\lambda$ ) there is an $I$-positive set $X$ such that $h \geq h_{\xi}$ on $X$ for $\lambda$ many $\xi$, but this contradicts the definition of $I$.

Using this claim, one can construct a $<_{I}$-increasing $\lambda$-sequence (a subsequence of $\left.\left\{h_{\xi}: \xi<\lambda\right\}\right)$ of functions that is $<_{I}$-cofinal in $\prod_{i \in S} g(i)$. Let $\lambda_{i}=\operatorname{cf} g(i)$, for each $i \in S$. The product $\prod_{i \in S} \lambda_{i}$ has a $<_{I}$-cofinal $<_{I^{-}}$ increasing sequence of length $\lambda$, and since $I$ is a normal ideal, we have $\lambda_{i}>\aleph_{\gamma_{i}}$ for $I$-almost all $i$. Now if $D$ is any ultrafilter extending the dual of $I, D$ satisfies $\operatorname{cf}\left(\prod_{i \in S} \lambda_{i} / D\right)=\lambda$.

Back to the proof of Lemma 4. For each $i \in S$ we have a regular cardinal $\lambda_{i}$ such that $\aleph_{\gamma_{i}}<\lambda_{i} \leq \aleph_{\gamma_{i}} \aleph_{1}$. By the assumption (6) we have $\aleph_{\gamma_{i}} \aleph_{1_{1}} \leq \aleph_{\gamma_{i}+\omega} \aleph_{0}$, 
and so $\lambda_{i} \leq \aleph_{\gamma_{i}+\omega} \aleph_{0}$. We use the following result:

Theorem (Shelah [ShA2], Chapter XIII, 5.1). Let $\aleph_{\delta}$ be such that $\aleph_{\delta}{ }^{\aleph_{0}}<\aleph_{\delta+\omega}$. Then for every regular cardinal $\mu$ such that $\aleph_{\delta}<\mu \leq \aleph_{\delta+\omega} \aleph_{0}$ there is an ultrafilter $U$ on $\omega$ such that $\operatorname{cf}\left(\prod_{n \in \omega} \aleph_{\delta+n} / U\right)=\mu$.

We apply the theorem to each $\aleph_{\gamma_{i}}$, and obtain for each $i \in S$ an ultrafilter $U_{i}$ on $\omega$ such that $\operatorname{cf}\left(\prod_{n \in \omega} \aleph_{\gamma_{i}+n} / U_{i}\right)=\lambda_{i}$. Combining the ultrafilters $U_{i}$ with the ultrafilter $D$ on $S$ from Lemma 9 we get an ultrafilter $U$ on the set

$$
A=\left\{\aleph_{\gamma_{i}+n}: i \in S, n=1,2, \ldots\right\}
$$

such that $\operatorname{cf}(\Pi A / U)=\lambda$. Hence $\lambda \in \operatorname{pcf}(A)$.

We shall now complete the proof of Lemma 4 by showing that $\max \operatorname{pcf}(A) \leq$ $\aleph_{\gamma+\omega}^{\aleph_{0}}$.

We have $A=\bigcup_{n=1}^{\infty} A_{n}$, where

$$
A_{n}=\left\{\aleph_{\gamma_{i}+n}: i \in S\right\}
$$

and since $2^{|A|}=2^{\kappa}<\min (A)$, we apply the corollary of Lemma 7 and get

$$
\max \operatorname{pcf}(A)=\max \operatorname{pcf} \bigcup_{n=1}^{\infty} s\left(\operatorname{pcf}\left(A_{n}\right)\right),
$$

where for each $n, s\left(\operatorname{pcf}\left(A_{n}\right)\right)$ is a finite subset of $\operatorname{pcf}\left(\operatorname{pcf}\left(A_{n}\right)\right)=\operatorname{pcf}\left(A_{n}\right)$.

Let $E=\bigcup_{n=1}^{\infty} s\left(\operatorname{pcf}\left(A_{n}\right)\right)$. Since (by Lemma 8) $\max \operatorname{pcf}\left(A_{n}\right) \leq \aleph_{\gamma+n}$ for each $n, E$ is a countable subset of $\aleph_{\gamma+\omega}$. Hence $\max \operatorname{pcf}(E) \leq \aleph_{\gamma+\omega} \aleph_{0}$, and so

$$
\lambda \leq \max \operatorname{pcf}(A)=\max \operatorname{pcf}(E) \leq \aleph_{\gamma+\omega}{ }^{\aleph_{0}} .
$$

\section{REFERENCES}

[GH] F. Galvin and A. Hajnal, Inequalities for cardinal powers, Ann. of Math. (2) 101 (1975), 491-498.

[M] M. Magidor, On the singular cardinals problem. I, Israel J. Math. 28 (1977), 1-31.

[ShA2] S. Shelah, Proper Forcing, Lecture Notes in Math., vol. 940, Springer-Verlag, 1982.

[Sh345] _ Products of regular cardinals and cardinals invariants of products of Boolean algebras, Israel J. Math. 70 (1990), 129-187.

[Sh355] _ , $\aleph_{\omega+1}$ has a Jonsson algebra, preprint.

[T] A. Tarski, Quelques théorèmes sur les alephs, Fund. Math. 7 (1925), 1-14.

Department of Mathematics, The Pennsylvania State University, University Park, PenNSYlvania 16802

Department of Mathematics, The Hebrew University, Jerusalem, Israel 